\section{CKD-MBD un caso "resistente": alcuni commenti}

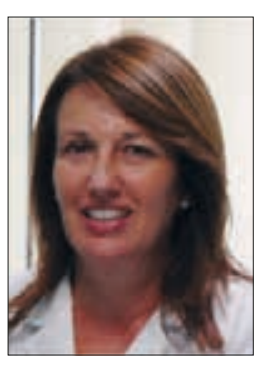

M. Cristina Mereu
Ringrazio l'Editor per la richiesta di un mio commento sul caso clinico pubblicato nel Vol. 24 no. 4 del Giornale di Tecniche Nefrologiche e Dialitiche del 2014 (1). Il caso, certamente interessante e non usuale, ci deve far ricordare che alcuni pazienti sono a elevato rischio di ipovitaminosi $D$ per varie cause (Tab. I) e, tra queste, figura l'uso di farmaci anticonvulsivanti (2). Numerosi studi hanno ormai dimostrato come i farmaci antiepilettici costituiscano il maggior fattore di rischio per l'instaurarsi di un'osteopatia metabolica caratterizzata da: osteopenia/osteoporosi, fratture, ipovitaminosi D e ipocalcemia (3). Infatti, come riportato in un recente articolo, già dopo 6 mesi di terapia i farmaci antiepilettici sono in grado di causare ipovitaminosi D nel $94 \%$ di un gruppo di pazienti (tra i 12 e i 65 anni) con precedenti normali livelli di vitamina (4). Il meccanismo di aumentata induzione del metabolismo della vitamina $D$ è stato esposto in modo chiaro ed esaustivo da Adriana Dusso et al. (5). Vorrei invece ricordare che fisiologicamente l'assorbimento intestinale del calcio è massimo solo a livelli di $25-(\mathrm{OH})$ D compresi tra 20 e $32 \mathrm{ng} / \mathrm{mL}$, mentre livelli $<20 \mathrm{ng} / \mathrm{mL}$ causano aumentata secrezione di Paratormone (PTH) fino all'instaurarsi di un quadro di iperparatiroidismo (IPT) se non si raggiungono livelli sierici di $30-40 \mathrm{ng} / \mathrm{mL}$ (6). Pertanto nella nostra pratica clinica dobbiamo sempre tenere presente che il deficit di vitamina $D$, che si instaura molto precocemente nella malattia renale cronica (MRC), ancora prima del $3^{\circ}$ stadio, va sempre corretto con le vitamine
D nutrizionali (D2, D3) e deve sempre costituire il primo step terapeutico per il controllo dell'IPTS in tutti gli stadi di malattia, anche in dialisi, e precedere l'uso delle forme attive di vitamina (VDRa agonisti selettivi e non selettivi). In questo paziente, giunto molto tardi all'osservazione del nefrologo, è presumibile che I'IPT fosse preesistente all'instaurarsi della MRC, la quale a sua volta non ha fatto altro che accelerarne l'evoluzione, mentre l'apparente resistenza terapeutica era causata ancora una volta dall'assunzione continua della carbamazepina che ha vanificato ogni tipo di terapia sia con calcitriolo che con paracalcitolo. La stessa paratiroidectomia non è stata risolutiva, perché non ha modificato l'andamento clinico e bioumorale dell'IPTS e pertanto, a mio parere, forse si poteva evitare. Infatti, è ormai accertato che l'iperstimolazione cronica delle paratiroidi induce proliferazione cellulare che dapprima comporta un'iperplasia diffusa con crescita policlonale accompagnata da una down-regulation dell'espressione di CaR e VDR e in seguito si trasforma in un'iperplasia nodulare con crescita monoclonale caratterizzata da assenza pressoché totale degli stessi recettori (7). Inoltre è stato dimostrato che la grandezza ghiandolare si correla con la forma istologica, per cui ghiandole con diametro complessivo $>1 \mathrm{~cm}$ e volume complessivo $>500 \mathrm{~mm}^{3}$ sono in genere considerate come ghiandole con degenerazione nodulare monoclonale, mentre quelle con diametro $<1 \mathrm{~cm}$ e volume $<500 \mathrm{~mm}^{3}$ sono da ritenersi ancora con iperplasia diffusa e pertanto suscettibili di terapia medica (8). Per questi motivi il volume ghiandolare è ritenuto uno dei fattori decisivi della severità dell'IPTS, che, insieme a persistenti ed elevati livelli di PTH (>800 pg/mL) e presenza di ipercalcemia e iperfosfatemia resistenti alla terapia medica, devono far orientare alla paratiroidectomia (PTX), come suggerito dalle Linee Guida K/DIGO 2009. L'ecografia con color-Doppler ad alta risoluzione ci consente di misurare le dimensioni e

TABELLA I - Cause di deficit di vitamina D (2)

- Ridotta sintesi cutanea: riduzione del 75\% con età >70 anni, minore esposizione ai raggi UV, razza nera.

- Ridotta biodisponibilità: malnutrizione, sindrome da malassorbimento, morbo celiaco, morbo di Chron, fibrosi cistica, malattia di Whipple, by-pass chirurgico.

- Obesità: maggiore sequestro di vitamina D nel grasso sottocutaneo.

- Aumentato catabolismo: farmaci anticonvulsivanti, corticosteroidei e barbiturici.

- Aumentata perdita di 25-idrossi vitamina D: sindrome nefrosica.

- Ridotta sintesi di 25-idrossi vitamina D: insufficienza epatica.

- Ridotta sintesi di 1.25-diidrossi vitamina D: tutti gli stadi dell'IRC.

- Disordini ereditari: varie forme di rachitismo congenito (mutazione del gene VDR, mutazione del CYP27B1, ecc.).

- Disordini acquisiti: iperparatiroidismo lº, sarcoidosi, tubercolosi e osteomalacia indotta da tumori. 
le variazioni volumetriche delle paratiroidi nel corso della loro "evoluzione morfologica". Nel caso in questione, però, la grandezza ghiandolare rilevata nell'unica paratiroide visibile $(0.7 \mathrm{~cm})$ presumibilmente era indicativa di una crescita ghiandolare policlonale e quindi di iperplasia diffusa suscettibile ancora di terapia medica. Un ultimo commento: la terapia con calciomimetico non dovrebbe mai essere intrapresa in pazienti con livelli sierici di calcemia (corretta per l'albumina) più bassi del limite inferiore del range di normalità $(<8.4 \mathrm{mg} / \mathrm{dL}$ ), poiché possono insorgere non solo convulsioni, ma anche prolungamento dell'intervallo QT, con rischio di aritmia. In conclusione, sono d'accordo con Adriana Dusso et al. sul fatto che, in questo paziente affetto da epilessia in terapia continua con carbamazepina da oltre 10 anni, il trattamento precoce con vitamina $D$ nativa, oltre che ristabilire i livelli vitaminici, forse avrebbe potuto evitare I'instaurarsi di un IPT e/o comunque avrebbe comportato una maggiore risposta alla terapia con calcitriolo e paracalcitolo, per up-regulation di VDR e CaSr, con conseguente aumento della calcemia, eventuale introduzione di calcimimetici, che probabilmente avrebbero evitato al paziente l'intervento di paratiroidectomia.

\section{Disclosures}

Financial support: No financial support was received for this submission.

Conflict of interest: The authors has no conflict of interest.

\section{Cristina Mereu}

Direttore U.O. Nefrologia e Dialisi, Ospedale NS di Bonaria, San Gavino Monreale (VS)
Indirizzo per la corrispondenza:

Dr.ssa M. Cristina Mereu

Direttore U.O. Nefrologia e Dialisi

Ospedale NS di Bonaria

Via Roma 1

09036 San Gavino Monreale (VS)

mcmereu@aslsanluri.it

\section{Bibliografia}

1. Polidoro M. CKD-MBD: un caso "resistente". Giornale di Tecniche Nefrologiche e Dialitiche 2014;26(4):348-53.

2. Holick MF. Vitamin D Deficiency. N Engl J Med 2007;357:266-81.

3. Teagarden DL, Meador KJ, Loring DW. Low vitamin D levels are common in patients with epilepsy. Epilepsy Res 2014;108(8): 1352-6.

4. Menon B, Harinarayan CV. The effect of anti epileptic drug therapy on serum 25-hydroxyvitamin $D$ and parameters of calcium and bone metabolism--a longitudinal study. Seizure 2010;19(3):153-8.

5. Dusso A, Arcidiacono MV. La carbamazepina antagonizza la soppressione di vitamina D-mediata dell'iperparatiroidismo secondario: patogenesi e possibilità terapeutiche. Giornale di Tecniche Nefrologiche e Dialitiche 2014;26(4):354-8.

6. Holick MF, Garabedian M. Vitamin D: photobiology, metabolism, mechanism of action, and clinical applications. American Society for Bone and Mineral Research 2006:129-37.

7. Tominaga Y, Sato K, Tanaka Y, Numano M, Uchida K, Takagi H. Histopathology and pathophysiology of secondary hyperparathyroidism due to chronic renal failure. Clin Nephrol 1995;44(Suppl. 1): S42-7.

8. Indridason OS, Heath H 3rd, Khosla S, Yohay DA, Quarles LD. Non suppressible parathyroid hormone secretion is related to gland size in uremic secondary hyperparathyroidism. Kidney Int 1996; 50:1663-7.

Published online: January 23, 2015 